\title{
Erratum to: A Heterologous Fibrin Glue Enhances the Closure Effect of Surgical Suture on the Repair of Annulus Fibrous Defect in a Sheep Model
}

\section{Zhi-cai DU ${ }^{1,2}$, Li-xin ZHU ${ }^{1}$}

${ }^{1}$ Department of Spinal Surgery, Orthopaedic Medical Center, Zhujiang Hospital, Southern Medical University, Guangzhou 510282, China

${ }^{2}$ Department of Minimally Invasive Spinal Surgery, The Second Affiliated Hospital of Inner Mon-golia Medical University, Hohhot 010030, China

(C) Huazhong University of Science and Technology 2020

Erratum to: Current Medical Science

DOI https://doi.org/10.1007/s11596-019-2079-2

The original version of this article unfortunately contained one mistake. The institutions of the authors are wrong. The corrected institutions are given below.

Zhi-cai DU ${ }^{1,2}$, Li-xin ZHU ${ }^{1}$

${ }^{1}$ Department of Spinal Surgery, Orthopaedic Medical Center, Zhujiang Hospital, Southern Medical University, Guangzhou 510282, China

${ }^{2}$ Department of Minimally Invasive Spinal Surgery, The Second Affiliated Hospital of Inner Mon-golia Medical University, Hohhot 010030, China 